LAWRENCE LIVERMORE NATIONAL LABORATORY
Position Estimation of Transceivers in Communication Networks

C. Kent, F. Dowla

January 14, 2004 
This document was prepared as an account of work sponsored by an agency of the United States Government. Neither the United States Government nor the University of California nor any of their employees, makes any warranty, express or implied, or assumes any legal liability or responsibility for the accuracy, completeness, or usefulness of any information, apparatus, product, or process disclosed, or represents that its use would not infringe privately owned rights. Reference herein to any specific commercial product, process, or service by trade name, trademark, manufacturer, or otherwise, does not necessarily constitute or imply its endorsement, recommendation, or favoring by the United States Government or the University of California. The views and opinions of authors expressed herein do not necessarily state or reflect those of the United States Government or the University of California, and shall not be used for advertising or product endorsement purposes.

This work was performed under the auspices of the U.S. Department of Energy by University of California, Lawrence Livermore National Laboratory under Contract W-7405-Eng-48. 


\title{
Position Estimation of Transceivers in Communication Networks
}

\author{
Claudia A. Kent and Farid U. Dowla \\ Lawrence Livermore National Laboratory \\ Livermore, CA 94550 USA
}

\begin{abstract}
With the rapid development in wireless sensor networks, there is an important need for transceiver position estimation independent of Global Positioning Systems (GPS) [1,3]. While GPS might be useful for outdoor sensor nodes, it is not for indoor node localization. In this case, position estimation is possible through network range estimates from time-of-flight (TOF) measurements, a technique well suited to large bandwidth physical links, such as in ultra-wideband (UWB) communications. For example, in our UWB systems, with pulse duration less than 200 pico-seconds, range can easily be resolved to less than a foot. Assuming an encoded UWB or spread spectrum physical layer, we developed algorithms and simulation tools to test transceiver position localization. Simulations were designed to lend insight into system characteristics such as position error sensitivities to network geometry, to range estimation errors, and to number of sensor nodes.
\end{abstract}

\section{INTRODUCTION}

In many sensor network applications, such as environmental monitoring of water in the soil or chemicals in the air, it is important to know the position of the network nodes. Range estimation from TOF data for a pair of communicating nodes is particularly attractive when using short-duration or high-frequency pulses such as UWB systems, and to a lesser extent for wireless local area network links in the 2.4 and $5 \mathrm{GHz}$ bands. For example, from radar theory, the root mean square (rms) range error in meters is given by [4]:

$$
\delta R \approx \frac{c}{B W \sqrt{S N R}}
$$

where $B W$ is the bandwidth of the pulse, $S N R$ is the signal to noise ratio at the receiver, and $c$ is the speed of light, $3 \times 10^{8} \mathrm{~m} / \mathrm{s}$. For bandwidths of $10 \mathrm{MHz}, 100 \mathrm{MHz}$, and $1 \mathrm{GHz}$ (corresponding approximately for 802.11b, 802.11a, and UWB systems), the rms range errors are $3 \mathrm{~m}, 0.3 \mathrm{~m}$ and $0.03 \mathrm{~m}$, respectively, for an assumed SNR of $20 \mathrm{~dB}$. We cannot expect to achieve this accuracy here, as we are using standard communication protocols and not dedicated radars, so we expect our range errors to increase one to two orders of magnitude. The range errors for an 802.11-a link can then be anywhere from 3 to 30 meters. On the other hand, we expect the more robust UWB systems to perform better than this. The wideband nature of the pulses allows us to determine the arrival times in a correlation filter more precisely, compared to narrow band systems. For example, in UWB systems developed at LLNL, the radio-frequency (RF) pulse duration is only about 200 pico-seconds. Hence, the arrival time of the pulses can resolved to less than a foot. In this work we assume an UWB network is used, and we simulate node position estimation for a set of communicating nodes. 
To achieve accuracy on the order of one foot, the transmitters and receivers each have orthogonal binary identification (ID) codes. A transmitter sends its encoded UWB signal to the receiver who replies with the exclusive-or of the transmitter and receiver codes. On receiving the reply, the transmitter extracts the receiver ID code and time-lapse information to calculate the range of the receiver node. Orthogonal codes provide unique radio identity allowing ranging to take place as accurately as possible within the physical layer and not relying on timing within the software.

This paper is a discussion of our simulation investigation on node positioning from TOF data. For the simulations a network consists of transmitter and receiver nodes distributed randomly in a $100 \mathrm{~m} \times 100 \mathrm{~m}$ area. Transmitters maintain a priori knowledge of position via satellites or some other predetermined method, receivers have unknown position. Transmitters determine receiver position through time-of-flight ranging and information sharing. We are interested in many aspects of this scenario, and through MATLAB simulation we propose to make statements on the relationship between ranging accuracy and position estimation accuracy, the improvement in position estimation with additional transmitting nodes, the benefit of using a "ranged" receiver node as a pseudo-transmitter, and the importance of network geometry. Interested readers are referred to our references for a more extensive survey of current research in this area.

\section{SIMULATION SOFTWARE}

When independent transmitter range measurements are combined to locate a receiver, what is the relationship between transmitter ranging accuracy and receiver position estimation accuracy? Does the position estimate improve with additional transmitters? Can a receiver that has been "located" act as a "pseudo-transmitter" to improve the position estimate of other receivers? Finally, what is the importance of network geometry?

To answer these questions, we developed a MATLAB GUI-based communication and simulation package for the user to generate virtual networks of transmitters and receivers, and to specify the error associated with the ranging transactions. The software simulates ranging, and it maintains the necessary communications infrastructure, measurement filtering, and information sharing algorithms that allow position estimation. After computing a position estimate, it records the error as the distance between the estimated and actual position. 


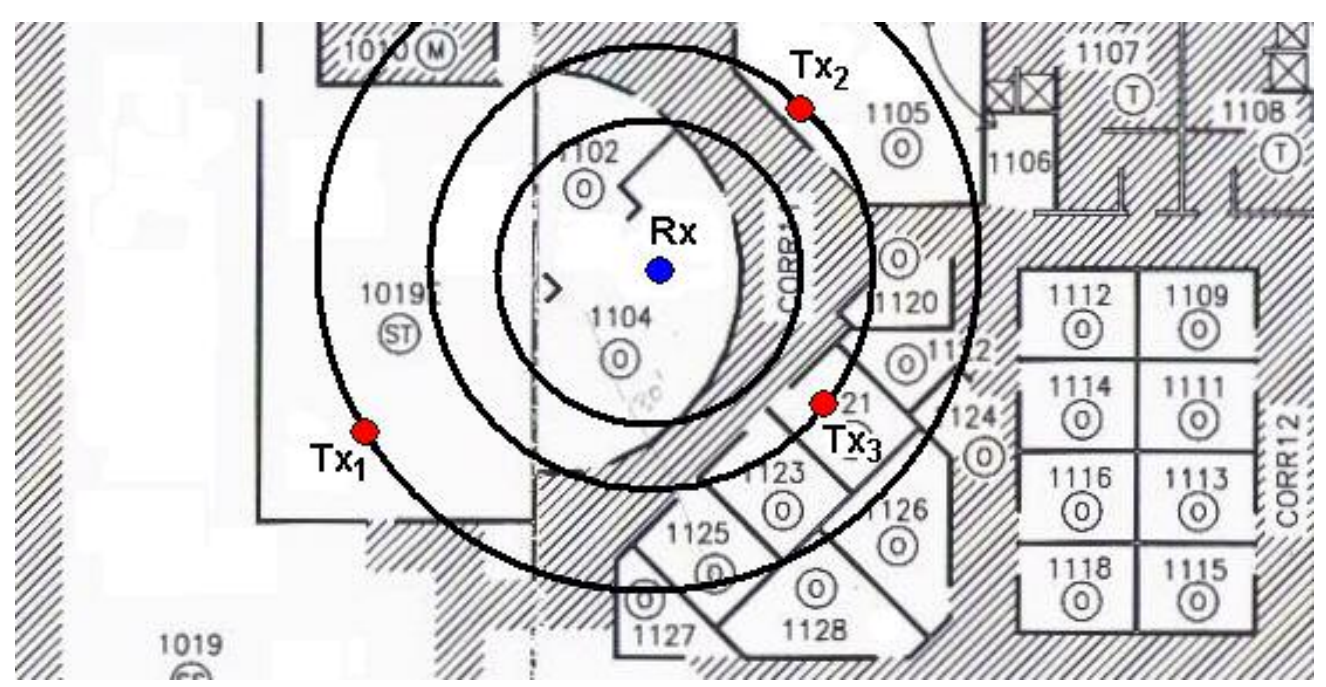

Figure 1. In the MATLAB GUI-based software the user designates a network of transmitters and receivers and simulates the network in ranging, filtering, data management, and position estimation.

\section{A. Range Measurement Error}

An UWB TOF range measurement will include error from several sources. Neither signal multi-path, nor receiver processing time can be predicted precisely. Rather than try, we model their effect as a uniformly distributed constant and assign to our simulated range measurements a uniformly distributed random measurement-bias within ranges of $\pm 5 \mathrm{ft}$., \pm $10 \mathrm{ft}$. , and so on.

The measurement-bias models the process error in a real system, and, we assume, a filter used eliminate the measurement-bias would also eliminate process error. We collect range measurements continuously and incorporate new measurements with those previous in a variation of a weighted least squares filter. The filter uses a set of measurements within a fixed length time window in a linear model that allows moving receivers, thus it takes into account both velocity and position. The weights for each measurement are their associated inverse variances. As each new measurement arrives, we calculate the new variance and find $R^{*}$, our bias-free range estimate, from the most recent set of measurements within our time window.

\section{B. Generating Position Estimates}

The software maintains a communications infrastructure to allow the transmitters to share their most current WLS-filtered range estimates, $R^{*}$, associated with each receiver. With enough $R^{* \prime}$ s, a position estimate is calculated using the closed-form method detailed in [5]. A graphical representation of the method is shown in Figure 2, where the $R^{*}$ measurements from two transmitters are combined in the Pythagorean Theorem (PT) to find receiver position. We combine the known transmitter positions, via GPS or some other positioning method, and the newly acquired $R^{*}$ measurements in multiple PT equations solved simultaneously to minimize equation error in a least-squares fashion. A minimum of 
three transmitters, and the corresponding three $\mathrm{R}^{*}$ measurements, is required for a unique receiver position. Two are shown in the figure, but a mirror triangle could be calculated placing the receiver outside of the concentric circles, thus three transmitters eliminate ambiguity.

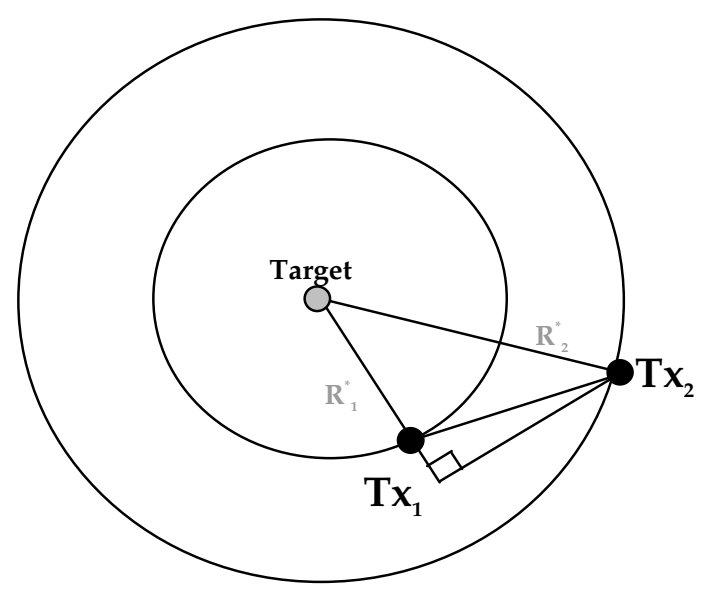

Figure 2. In this graphical representation of the closed-form least squares position estimation method developed in [1], the range measurements from multiple transmitters are combined using the Pythagorean Theorem for an estimate of position.

\section{RESULTS}

During a simulation, the position estimate of a receiver typically converges to and remains at a settled value after 1000 timesteps (one minute of sampling at 10ms). To insure convergence, we run all simulations for approximately 3000 timesteps. We generate hundreds of random networks for each experiment, and we take the final, converged value as the position error associated with the network.

\section{A. Ranging Accuracy and Additional Transmitters}

To measure the effect of additional transmitters on receiver position error, we generate 100 random networks of the minimum size, three transmitters and one receiver, and we simulate each with a small uniformly distributed range measurement error $( \pm 20 \mathrm{ft}$.). We then calculate the average and standard deviation of the converged values across all of the 100 networks and repeat the test while varying the number of transmitters from three through nine. The results are compiled in the errorbar plot of Figure 3 with mean position error and standard deviation as a function of number of transmitters. By increasing the number of transmitters to four, with this small range measurement error, mean position error decreases by nearly 20 feet, and measurement confidence increases (with a standard deviation decrease) by nearly $60 \mathrm{ft}$. The same benefit is not found by increasing the number of transmitters to five, where results show there is little improvement beyond four transmitters. 


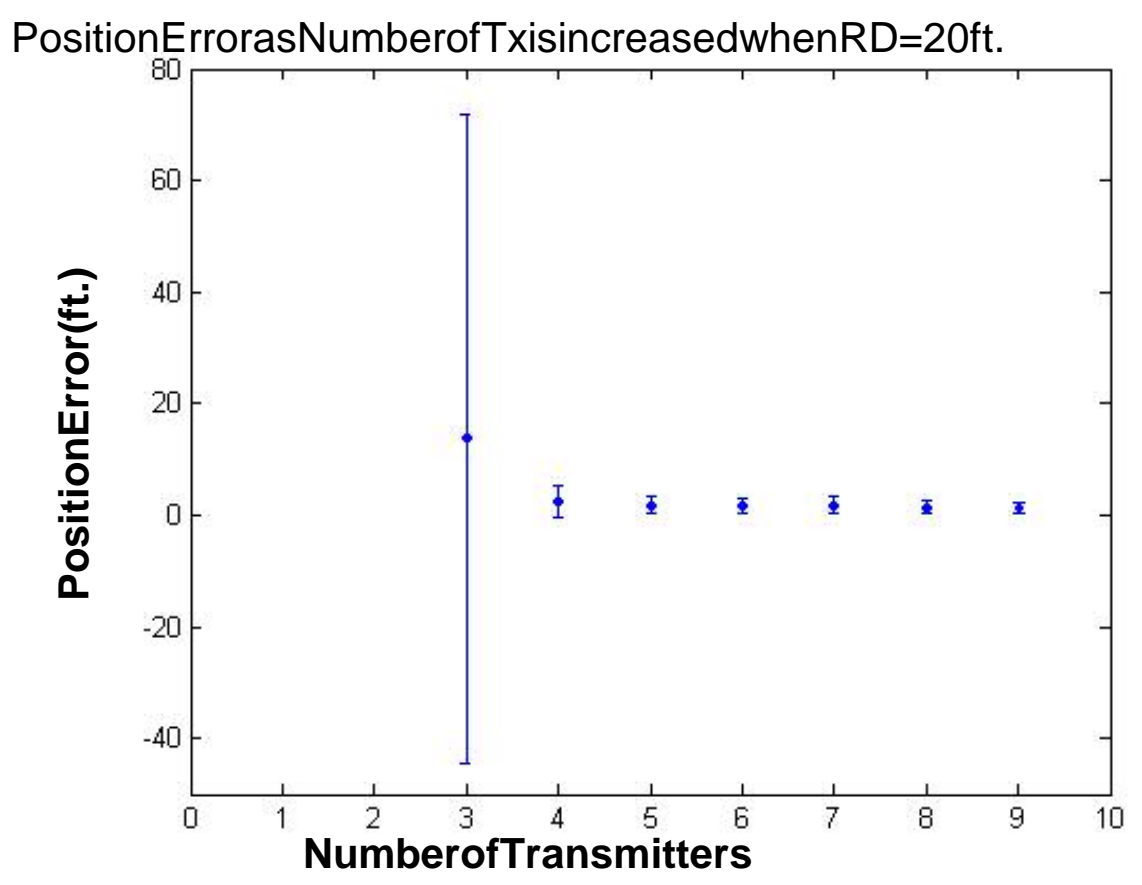

Figure 3. The mean and mean + STD were collected for networks ranging from three to nine transmitters and one receiver. All networks assumed a $\pm 20 \mathrm{ft}$. range measurement error. Four transmitters dramatically reduce both mean and standard deviation.

In Figure 3 the range measurement error is centered between $\pm 20 \mathrm{ft}$., and four transmitters provide optimal position accuracy. In Figure 4 we present data collected by varying range measurement error along with number of transmitters to find an overall correlation between the three. Confidence in four transmitters, rather than three, is valid only when range measurement error is kept below $\pm 60 \mathrm{ft}$. Above this, additional transmitters are necessary. 


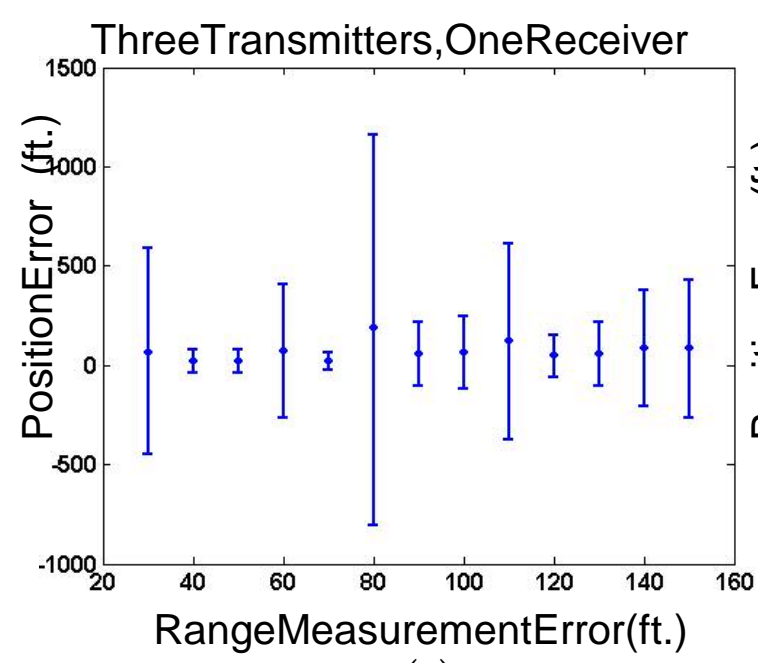

(a)

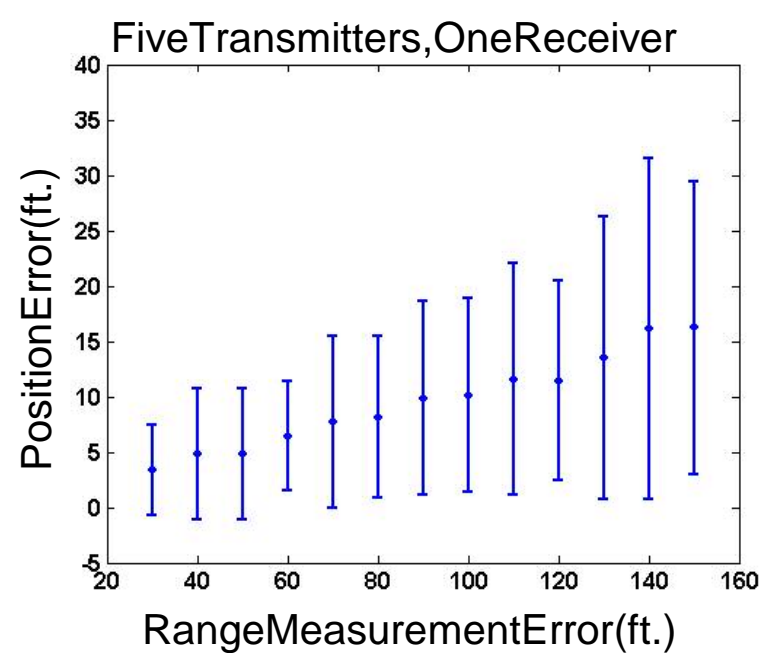

(c)

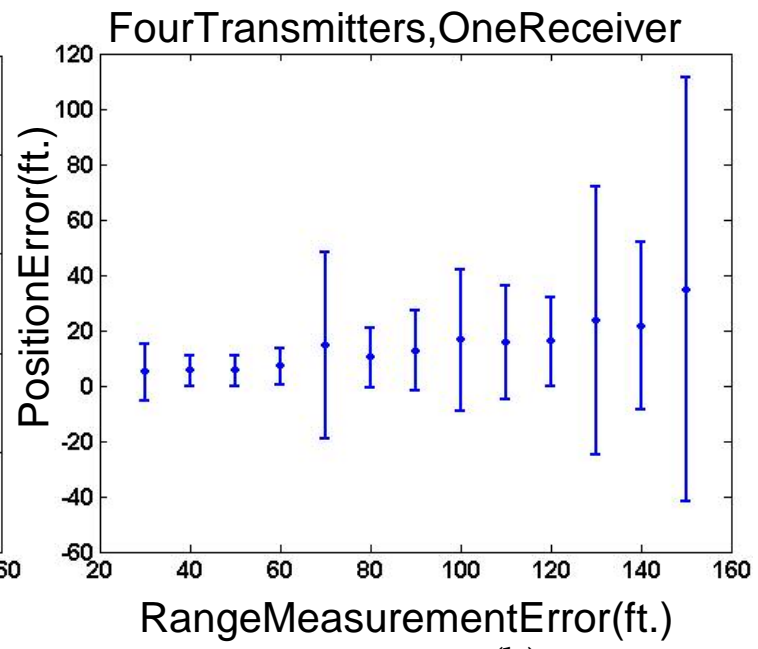

(b)

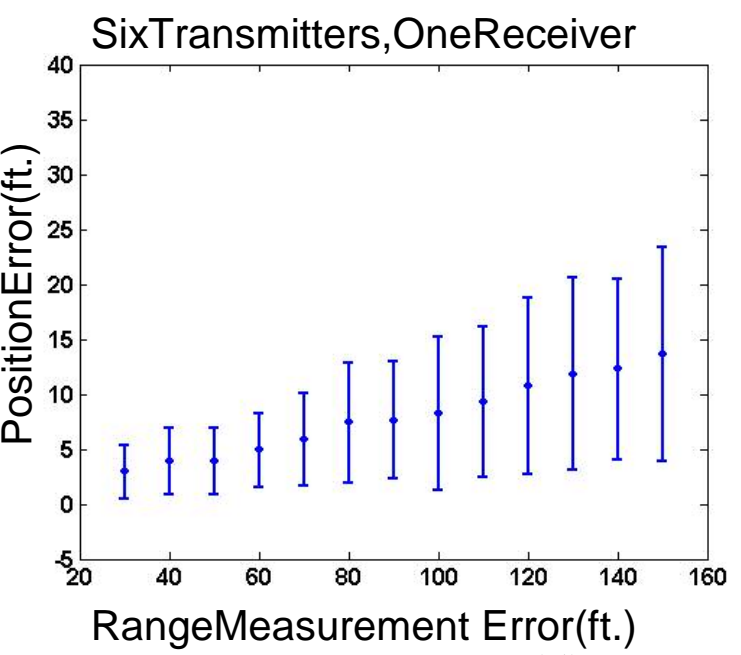

(d)

Figure 4. We are interested in the effect additional transmitters, but also the effect of an increased range measurement error. These errorbar plots of mean and standard deviation show the impact on position error by varying both of these factors with (a) three transmitters, one receiver, (b) four transmitters, one receiver, (c) five transmitters, one receiver, (d) six transmitters, one receiver.

\section{B. Pseudo-Transmitters}

Once a receiver has been "located," it can, theoretically, be used to improve the position estimate of another receiver and thus be considered a pseudo-transmitter. In this case, there is no difference between a transmitter and receiver (save the three dedicated transmitters needed for location and orientation reference). We test this using $N$ real transmitters and $M$ pseudo-transmitters, and we find that pseudo-transmitters do not improve the position estimate of a receiver as real transmitters do; instead, they introduce an undamped oscillation that worsens with additional pseudo-transmitters. We test this by varying $N=$ $[3, \ldots, 6]$ and $M=[1, \ldots, 6]$ and find all cases similar to that shown in Figure 5 where $N=5$ and $\mathrm{M}=[1, \ldots, 4]$. As the number of pseudo-transmitters increases, so does position error. The 
pseudo-transmitter does add knowledge to the system - without error in the range measurements, pseudo-transmitters would certainly improve the position estimate. However, with the slightest amount of error (here $\pm 10 \mathrm{ft}$.) the system becomes unstable.

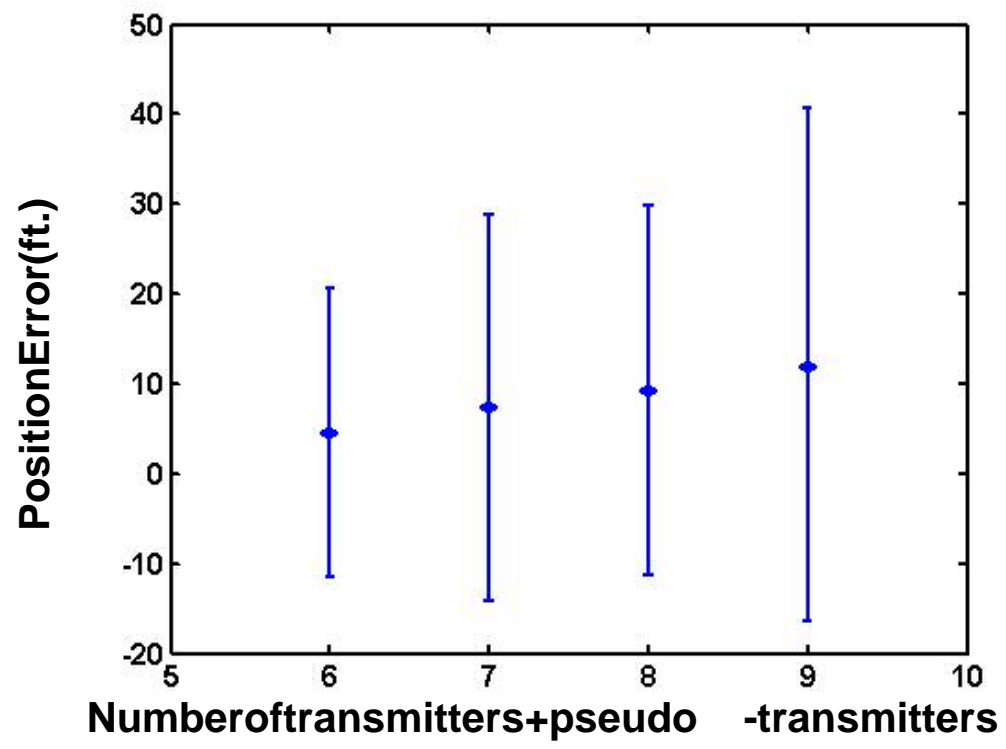

Figure 5: We use five transmitters and vary the number of pseudo-transmitters to show that pseudo-transmitters add instability to the system in the presence of external error, here it is a 10 foot range measurement error.

\section{Network Geometry}

Intuitively, transmitters can resolve the position of a centrally located receiver more accurately than one that is on the "outskirts." Since our simulations require thousands of networks, it is not practical to assign receivers manually, thus receivers and transmitters are randomly scattered across a $100 \mathrm{~m} \times 100 \mathrm{~m}$ region for all of our simulations. To verify the importance of receiver location within the network, we have created a non-dimensional number associated with each randomly scattered network that represents the receiver location within the transmitters. A geometric factor is defined as the receiver's distance from the geometric center-of-mass of the transmitters, normalized by the mean distance between transmitters. For a network confined to $100 \mathrm{~m} \times 100 \mathrm{~m}$, a low geometric factor $(<0.5)$ represents a "centrally located" receiver within a "closely spaced" network. 


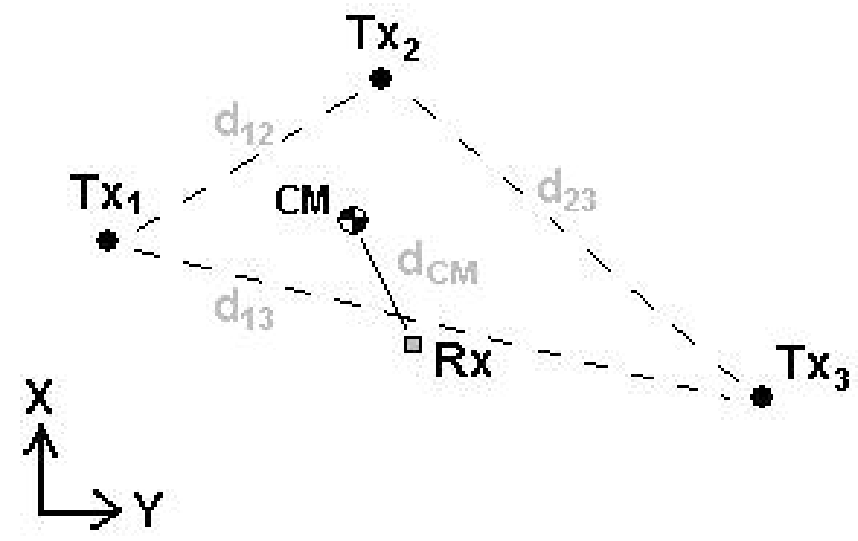

Figure 6. We took the receiver's distance to the center-of-mass of the transmitters, normalized it, and called it the geometric factor associated with each of our random networks.

For each of the random networks we record the geometric factor with position error and the results are in Figure 7. We confirm a correlation and propose that when the network geometric factor is less than approximately 0.5 the standard deviation of position error is within orders of magnitude of its mean.

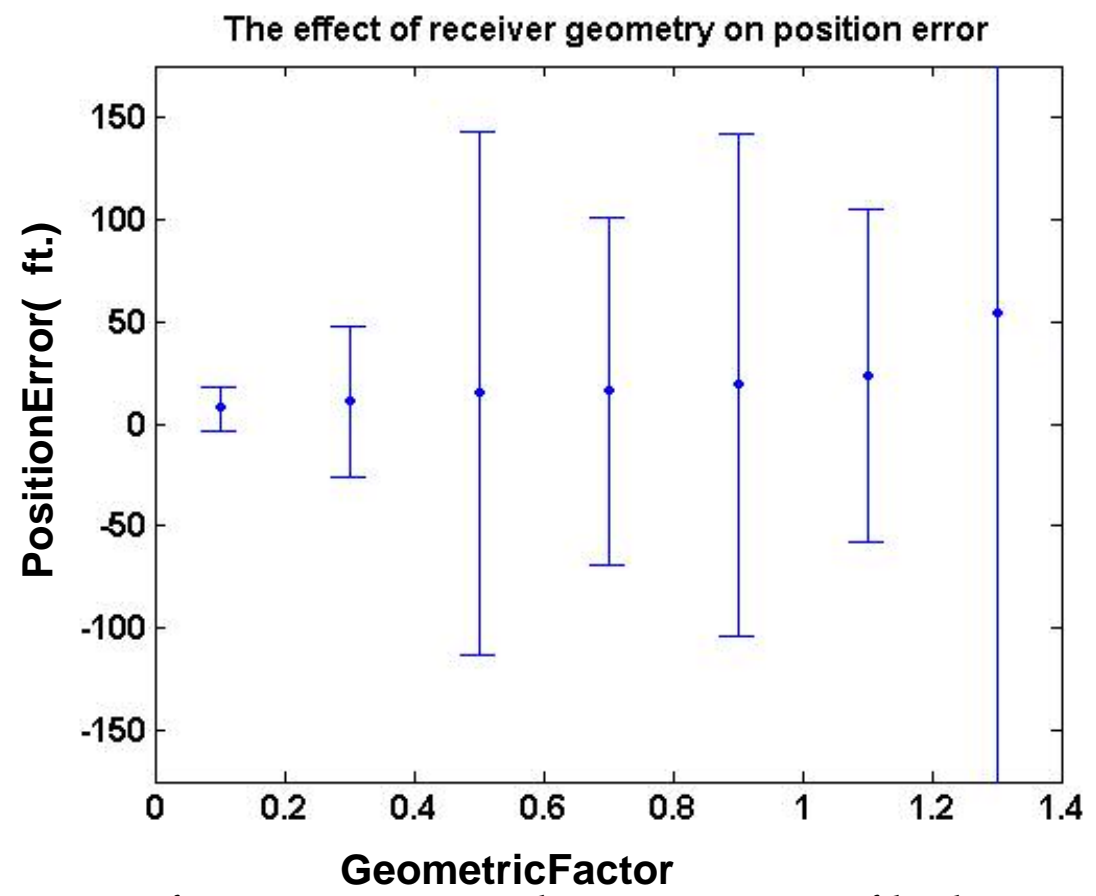

Figure 7. The geometric factor is a numerical representation of both transmitter density and receiver proximity. A "centrally located" receiver within a "closely spaced" network will have a geometric factor of $\sim<0.5$, and a low geometric factor implies confidence in a low position estimation error.

\section{CONCLUSION}

Our research has been successful in not only uncovering answers to our initial 
questions, but also laying the foundation necessary to implement our algorithms using recently available UWB radio hardware. Our MATLAB software package runs smoothly and is easy to use. We have tested thousands of random networks without algorithm error, and data collected from these tests has led to interesting insights. Four transmitting nodes in a network, rather than three, considerably improve the position estimate of a receiver. When operating with a $\pm 10 \mathrm{ft}$ range measurement error they average a position estimate accurate to within $3 \mathrm{ft}$. Above four, however, there is little improvement. A receiver's placement within the transmitters does affect its ability to be located, and using receivers as pseudotransmitters does not improve the position estimate for other receivers, as originally predicted. We have also quantified these dependencies. Finally, algorithms developed in this project have been successfully implemented and tested on IEEE 802.11a WLAN equipped laptop computers for a related wireless testbed project.

\section{ACKNOWLEDGEMENTS}

This research was supported through an internal Engineering Technology Development program, Lawrence Livermore National Laboratory. The authors would like to thank Steve Azevedo, Deputy Division Leader for the EETD, for his support of the project. This work was performed under the auspices of the U. S. Department of Energy by the University of California, Lawrence Livermore National Laboratory under Contract No. W-7405-Eng-48.

\section{REFERENCES}

[1] N. Bulusu, J.Heidemann, and D. Estrin, "GPS-less low cost outdoor localization for very small devices," IEEE Personal Communications Magazine, vol. 7, no. 5, pp. 28-34.

[2] F. Dowla, A. Spiridon, D. Benzel, T. Rosenbury. "Ultra-wideband Communication," FY02 Engineering technology Reports, Vol. 2, Laboratory Directed Research and Development, UCRL53868-02, pp. 4, 2003.

[3] D. Estrin, L.Gilrod, G. Potie, and M. Srivastava, "Instrumenting the world with wirelesss sensor networks," in Proc. IEEE Int. Conf. Acoustics, Speech, Signal Processing, vol. 4, pp. 2033-2036, Salt Lake City, Utah, USA May 2001.

[4] M, Skolnok, "Introduction to Radar Systems," McGraw-Hill Book Co., 1962, pp. 468-470.

[5] J. Smith and J. Abel, "Closed-Form Least-Squares Source Location Estimation from RangeDifference Measurements," IEEE Transactions on acoustics and Speech, vol. ASSP-35, no. 12, pp. 1661-1669, 1987.

[6] M. Uchida, et., al., "A Vehicle-to-Vehicle Communication and Ranging System Based on Spread Spectrum Technique," IEEE Vehicle Navigation \& Information Systems Conference Proceedings, 1994. 